\title{
Epsins 1 and 2 promote NEMO linear ubiquitination via LUBAC to drive breast cancer development
}

\author{
Kai Song, ${ }^{1}$ Xiaofeng Cai, ${ }^{1,2}$ Yunzhou Dong, ${ }^{1}$ Hao Wu, ${ }^{1}$ Yong Wei, ${ }^{3,4}$ Uma T. Shankavaram, ${ }^{5}$ Kui Cui, ${ }^{1}$ Yang Lee, ${ }^{1}$ Bo Zhu, \\ Sudarshan Bhattacharjee, ${ }^{1}$ Beibei Wang, ${ }^{1}$ Kun Zhang, ${ }^{1}$ Aiyun Wen, ${ }^{1}$ Scott Wong, ${ }^{1}$ Lili Yu, ${ }^{1}$ Lijun Xia, ${ }^{2}$ Alana L. Welm, \\ Diane R. Bielenberg, ${ }^{1}$ Kevin A. Camphausen, ${ }^{5}$ Yibin Kang, ${ }^{3,4}$ and Hong Chen ${ }^{1}$
}

'Vascular Biology Program, Boston Children's Hospital, Harvard Medical School, Boston, Massachusetts, USA. ²ardiovascular Biology Research Program, Oklahoma Medical Research Foundation, Oklahoma City, Oklahoma, USA. 'Department of Molecular Biology, Princeton University, Princeton, New Jersey, USA. ${ }^{4}$ Cancer Metabolism and Growth Program, Rutgers Cancer Institute of New Jersey, New Brunswick, New Jersey, USA. ${ }^{5}$ Radiation Oncology Branch, National Cancer Institute, Bethesda, Maryland, USA. ${ }^{6}$ Department of Oncological Sciences, University of Utah, Salt Lake City, Utah, USA.

\begin{abstract}
Estrogen receptor-negative (ER-negative) breast cancer is thought to be more malignant and devastating than ER-positive

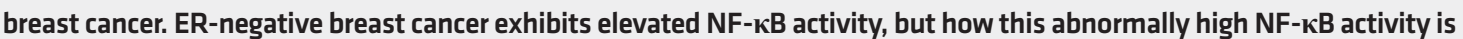
maintained is poorly understood. The importance of linear ubiquitination, which is generated by the linear ubiquitin chain assembly complex (LUBAC), is increasingly appreciated in NF-KB signaling, which regulates cell activation and death. Here, we showed that epsin proteins, a family of ubiquitin-binding endocytic adaptors, interacted with LUBAC via its ubiquitininteracting motif and bound LUBAC's bona fide substrate NEMO via its N-terminal homolog (ENTH) domain. Furthermore, epsins promoted NF-KB essential modulator (NEMO) linear ubiquitination and served as scaffolds for recruiting other components of the ІкB kinase (IKK) complex, resulting in the heightened IKK activation and sustained NF- $\kappa B$ signaling essential for the development of ER-negative breast cancer. Heightened epsin levels in ER-negative human breast cancer are associated with poor relapse-free survival. We showed that transgenic and pharmacological approaches eliminating epsins potently impeded breast cancer development in both spontaneous and patient-derived xenograft breast cancer mouse models. Our findings established the pivotal role epsins played in promoting breast cancer. Thus, targeting epsins may represent a strategy to restrain NF- $\kappa B$ signaling and provide an important perspective into ER-negative breast cancer treatment.
\end{abstract}

\section{Introduction}

$\mathrm{NF}-\kappa \mathrm{B}$ is a crucial oncogenic transcription factor that is frequently activated in estrogen receptor-negative (ER-negative) breast tumor subtypes $(1,2)$ and plays an obligatory role in breast cancer tumorigenesis and development $(3,4)$. Despite its heightened activity in breast cancer cells, NF- $\kappa \mathrm{B}$ signaling is further enhanced in response to elevated tumor-associated proinflammatory stimuli, including TNF- $\alpha$, leading to expression of downstream target genes to promote progression of breast cancer $(5,6)$. Stimulus-dependent NF- $\mathrm{B}$ activation occurs predominantly through I $\mathrm{B}$ kinase-dependent (IKK-dependent) phosphorylation and subsequent degradation of the NF- $\kappa$ B inhibitor I $\mathrm{B}$ (7). The IKK complex consists of the catalytic subunits, IKK- $\alpha$ and IKK- $\beta$, and the regulatory IKK- $\gamma$ subunit, also called NF- $\kappa \mathrm{B}$ essential modulator (NEMO) (8-10). Lys63-linked or linear polyubiquitination of NEMO plays a critical role in $\mathrm{NF}-\kappa \mathrm{B}$ activation in response to proinflammatory stimuli (11-14). It has been postulated that polyubiquitination may induce a conformational change in NEMO that is responsible for IKK complex assembly and NF- $\mathrm{BB}$ activation

Authorship note: KS, XC, YD, and HW contributed equally to this work Conflict of interest: The authors have declared that no conflict of interest exists. Copyright: ( 2021, American Society for Clinical Investigation.

Submitted: May 6, 2019; Accepted: September 17, 2020; Published: January 4, 2021 Reference information: / Clin Invest. 2021;131(1):e129374.

https://doi.org/10.1172/JCl129374.
(15-19). Given that NF- $\mathrm{BB}$ is thought to be constitutively activated in ER-negative breast cancer cells, the question of how NEMO polyubiquitination and therefore $\mathrm{NF}-\kappa \mathrm{B}$ activity are modulated in breast cancer needs to be answered.

Epsins are a family of ubiquitin-binding adaptor proteins with emerging importance in human diseases (20-26). Mammals express 3 epsins encoded by 3 genes (EPN1, -2, and -3) (20, 27, 28). Epsin 3 is highly expressed primarily in the stomach and wounded epithelia and is abundant in human gastric tumors $(25,27)$; epsins 1 and 2 are ubiquitously expressed and redundant in function (20, 28). As such, a single deletion of either epsin 1 or epsin 2 does not affect normal physiology; however, global double deletion of epsins 1 and 2 results in embryonic lethality at midgestation, phenotypically resembling mice deficient in the linear ubiquitin chain assembly complex (LUBAC), HOIL-1, and HOIP $(28,29)$. To circumvent embryonic lethality, we previously generated a conditional epsin 1-KO mouse on an epsin 2-null background. Whereas postdevelopmental epsin depletion in these mice did not produce gross abnormalities and resulted in normal survival under physiological conditions, specific loss of epsins 1 and 2 in endothelial cells inhibited tumor growth due to disruption in tumor angiogenesis $(24,30)$. These findings suggested that epsins play a key procancerous role in the tumor microenvironment. However, the tumor cell-intrinsic role of epsins is largely unexplored.

Our analyses revealed that epsins are upregulated in breast cancer relative to normal tissues (Supplemental Figure 1, A and B; 
supplemental material available online with this article; https:// doi.org/10.1172/JCI129374DS1), and high levels of epsins were associated with a low rate of relapse-free survival in all types of breast cancer patients - particularly ER-negative subtypes (Supplemental Figure 1C). Epsin proteins decode the ubiquitination signals from plasma membrane proteins to promote their clathrin-dependent endocytosis, resulting in the translocation of both the ubiquitinated cargo and epsins to endocytic degradative compartments, such as lysosomes $(24,31-34)$. In this study, we discovered that opposed to their trademark function as a degradation accelerator and receptor signaling attenuator for plasma membrane ubiquitinated receptors, epsins facilitate TNFR1 signaling by enhancing NEMO (IKK- $\gamma$ ) ubiquitination. Given that epsins are prominent ubiquitin-binding proteins and that NEMO is heavily ubiquitinated and its polyubiquitination is required for subsequent NF- $\kappa \mathrm{B}$ activation, we hypothesize that elevated epsin levels in breast cancer cells may potentiate $\mathrm{NF}-\kappa \mathrm{B}$ signaling through its interaction with NEMO and ubiquitin E3 ligases, including LUBAC, which are components involved in the canonical TNFR1 signaling (whose expression is detected predominantly in ER-negative versus ER-positive breast tumors) (35) (Supplemental Figure 1B), propelling ER-negative breast cancer development.

\section{Results}

To test our hypothesis, we established an MMTV-PyMT (estrogen-independent) spontaneous breast cancer mouse model with inducible global deletion of epsins by first crossing Epn1-floxed, Epn2-null $\left(E p n 1^{f / f l} E p n 2^{--}\right)$mice (24) with tamoxifen-inducible $E R^{T 2}$-Cre deleter mice, and then crossing the Epn $1^{f / f l} E p n 2^{-/-} E R^{T 2}$-Cre mice with $M M T V-P y M T$ mice that were bred onto the C57BL/6 background (Supplemental Figure 1D and Methods) to obtain iDKO/PyMT mice. In addition to WT MMTV-PyMT mice on the C57BL/6 background, we also utilized Epn1 ${ }^{+/+} E p n 2^{-/-} E R^{T 2}-C r e ~ M M T V-P y M T$ and Epn1 $1^{t / f l}$ Epn2 ${ }^{-/-}$MMTV-PyMT littermate mice as controls (Ctrl/PyMT). These mice displayed phenotypes that were indistinguishable from those of $W T / M M T V-P y M T$ mice, thus reinforcing the functional redundancy of epsins 1 and 2. WT/MMTV-PyMT or Ctrl/PyMT and iDKO/PyMT mice were administered 4-hydroxytamoxifen at 6 weeks of age and bypassed the initial mammary ductal development (Supplemental Figure 1, D-F). We observed that iDKO/PyMT mice exhibited dramatic reductions in primary mammary tumor burden compared with the control mice (Figure 1, A and B). Remarkably, iDKO/PyMT mice as early as 9 weeks of age exhibited significantly fewer and smaller tumor masses visualized by carmine red wholemount staining of inguinal mammary glands (Figure 1A), suggesting that epsins possess protumorigenic propensity. To specifically assess the tumor-intrinsic role of epsin in breast cancer formation, we created a constitutive mammary epithelium-specific epsindeficient $M M T V$-PyMT mouse model by sequentially crossing Epn $1^{f / / f l}$ Epn2 ${ }^{-1-}$ mice with $M M T V-C r e$ deleter mice and $M M T V-P y M T$ mice on the C57BL/6 background to obtain $M E p C-D K O / P y M T$ mice (Supplemental Figure 1, G-I and Methods). Likewise, in addition to $W T / M M T V-P y M T$ mice on the C57BL/6 background, we also

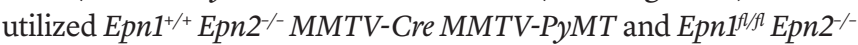
$M M T V-P y M T$ littermate mice as controls because these mice displayed phenotypes that were indistinguishable from those of WT/ $M M T V-P y M T$ mice. Similar to the $i D K O / P y M T$ mice, $M E p C-D K O /$
PyMT mice exhibited smaller tumor masses as early as 9 weeks of age (Figure 1C), reduced tumor growth, and decreased overall primary tumor burden during weeks 12 to 24 (Figure 1D) relative to the control mice. In addition, specific and efficient epsin deletion (Figure $2 \mathrm{E}$ ) in mammary epithelial cells prevented early breast cancer onset without disturbing the primary mammary ductal outgrowth in MMTV-PyMT mice, as evidenced by whole-mount staining of inguinal mammary glands harvested from 4-, 6-, 9-, and 11-week-old $M E p C-D K O$ mice versus age-matched littermate controls (Figure 1C and data not shown), reflecting a cancer cell-specific positive regulatory role epsins play in breast cancer development in mice.

To investigate whether epsins equivalently promote breast cancer development in humans, we generated a stable epsindeficient human breast cancer cell line by infecting ER-negative MDA-MB-231 (MDA231) cells with lentivirus containing shRNAs specific for epsins 1 and 2 (Supplemental Figure 1J). Orthotopically implanted epsin-deficient MDA231 cells impeded primary tumor growth (Figure 1E) in nude mice. Similarly, s.c. implanted epsin-deficient MDA231 cells produced fewer tumors relative to the control cells in nude mice (Figure 1F). Collectively, these data suggested that epsins were sufficient and necessary to promote human breast cancer development.

To explore the protumorigenic mechanisms modulated by epsins, the Cancer Genome Atlas Breast Invasive Carcinoma (TCGABRCA), a large-scale data set, was employed to analyze the pathways most related to elevated levels of epsins in human breast cancer. Among other well-documented pathways, such as Notch (28), VEGF $(24,30,36,37)$, and Wnt (26), gene set enrichment analysis (GSEA) uncovered a role for epsins as a potentially novel regulator of NF- $\mathrm{BB}$ in breast cancer (Supplemental Figure 2, A and B). Furthermore, gene expression microarray analysis of control and epsin-deficient MDA231 cells revealed that epsin deficiency markedly reversed the enrichment of multiple NF- $\kappa B-$ related gene sets (Table 1). Leading-edge analysis of the GSEA results identified $178 \mathrm{NF}-\kappa \mathrm{B}$ signature genes that were significantly downregulated ( $>1.3$-fold change) upon epsin loss. Among them, 21 genes presented in at least 3 signatures exhibited a greater than 2 -fold change in expression (Table 2).

Importantly, our observation that $\mathrm{p}-\mathrm{I} \kappa \mathrm{B}-\alpha$ levels in human breast cancer tissues were elevated compared with adjacent normal tissue (data not shown) supports the role of constitutive NF- $\kappa \mathrm{B}$ activation in breast cancer. Given the newly identified connection of epsin and NF- $\kappa \mathrm{B}$ activation in breast cancer in our GSEA, we sought to examine whether epsin directly controls NF- $\kappa B$ activation by using an EMSA assay (38). Epsin deficiency weakened constitutive p65/p50 NF-kB DNA binding activity that was prominent in control MDA231 cells (Figure 2A, lanes 2 and 3, and Supplemental Figure $2 \mathrm{C}$ ), reflecting the crucial role epsins play in facilitating $\mathrm{NF}-\kappa \mathrm{B}$ activation. Accordingly, epsin deficiency significantly reduced the activation of the constitutive NF- $\kappa \mathrm{B}$ pathway, including phosphorylation of IKK- $\alpha / \beta$, I $\mathrm{B}-\alpha$, and p65 in MDA231 (Figure $2 \mathrm{~B}$ and Supplemental Figure 2D). Likewise, epsin deficiency significantly hindered phosphorylation of p65 NF- $\kappa \mathrm{B}$ and/or I $\mathrm{BB}-\alpha$ in tumors from $M E p C-D K O / P y M T$ mice (Figure 2, $\mathrm{C}$ and $\mathrm{D}$ ) and in orthotopic MDA231 xenografts (Supplemental Figure 2E). Using a converse approach, we found that overexpression of epsin 1 in the noncancerous human breast epithelial cell line MCF10A increased nuclear translocation of $\mathrm{p} 65$, a hallmark of NF- $\mathrm{BB}$ activation, and the 
A

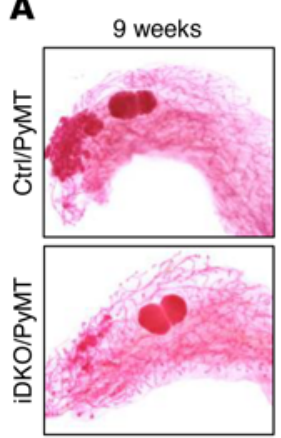

C
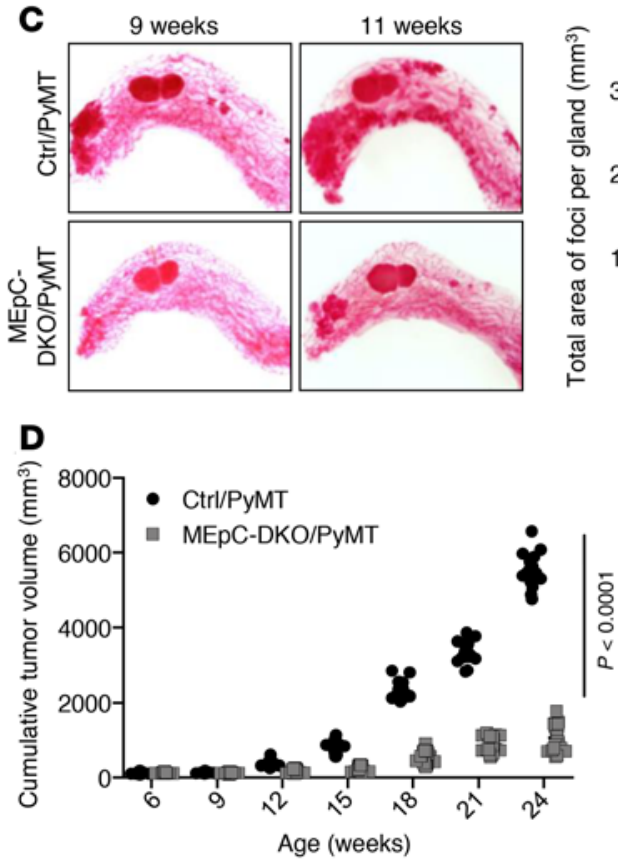

E

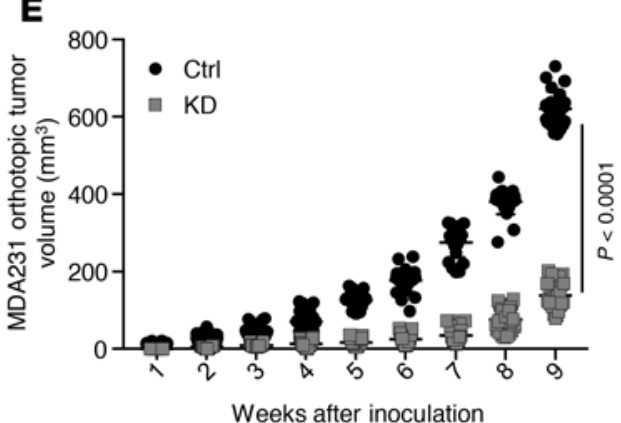

B
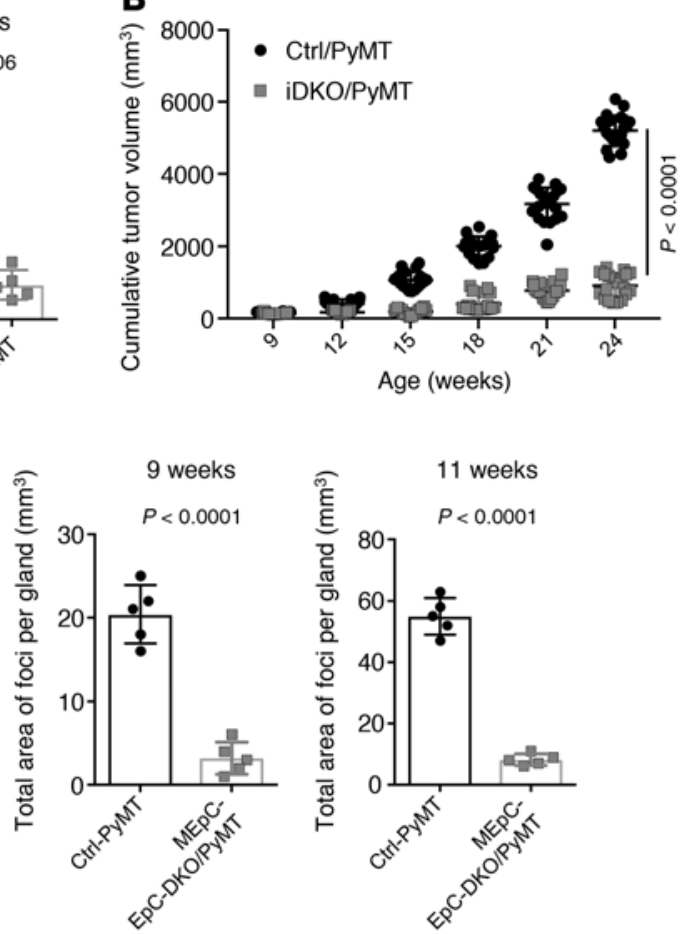

$\mathbf{F}$

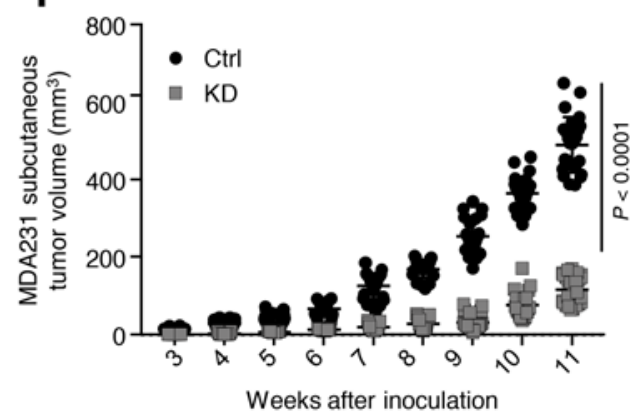

Figure 1. Epsin plays a procancerous role in spontaneous mouse mammary tumor models and human breast cancer xenograft models. (A) Whole-mount staining and quantification of tumor lesions in inguinal mammary glands from control MMTVPyMT mice (Ctrl; see description in Results) and MMTV-PyMT with global inducible deletion of epsins 1 and 2 (iDKO) mice at 9 weeks of age ( $n=5$ mammary glands per genotype). (B) Growth curves of mammary tumors in Ctrl/PyMT and iDKO/PyMT mice ( $n=20$ per genotype). (C) Wholemount staining and quantification of tumor lesions in inguinal mammary glands from MMTV-PyMT mice (Ctrl) and MMTV-PyMT with mammary epithelium-specific deletion of epsins 1 and 2 (MEpC-DKO, see description in Results) mice at 9 and 11 weeks ( $n=5$ mammary glands per genotype). (D) Growth curves of mammary tumors in Ctrl and MEpC-DKO MMTV-PyMT mice ( $n=20$ per genotype). (E) Growth curves of orthotopically implanted Ctrl (generated using lentivirus-containing scrambled shRNA sequences of epsins 1 and 2) or epsin 1/2 knockdown (KD) MDA231 tumors in nude mice ( $n=25$ per genotype). (F) Growth curves of s.c. implanted Ctrl or KD MDA231 tumors in nude mice $(n=25$ per genotype). Statistical values were calculated using an unpaired, 2-tailed $t$ test (A and $\mathbf{C}$ ) or 2-way ANOVA followed by Sidak's multiple-comparisons test (B and $\mathbf{D}-\mathbf{F}$ ). Data are presented as mean $\pm \mathrm{SD}$. See also Supplemental Figure 1. expression of NF- $\mathrm{BB}$ target genes, CXCL1, CXCL3, IL8, IL1b, and TNFa (Supplemental Figure 2F). ER-positive MCF7 breast cancer cells with stably enforced epsin 1 expression exhibited heightened p-p65 and p-IКB- $\alpha$, and SCID mice s.c. injected with these cells displayed significantly larger primary tumors, compared with control MCF7 cells (Supplemental Figure 2G). Additionally, we identified a strong positive correlation of epsin 1 expression level with nuclear localization of p-p65 by immunostaining of a human breast tissue array (Figure 2, E and F). Furthermore, GSEA of the TCGA-BRCA data set showed that combined expression of EPN1 and EPN2 positively correlated with those of NF- $\kappa \mathrm{B}-$ related genes, including NEMO (IKBKG), IKK- $\alpha$ (CHUK), and Iк-B (NFKBIA and NFKBIB), in human breast cancer (Figure $2 G$ ). These findings indicate that high expression levels of epsins in breast cancer are responsible, at least in part, for elevated constitutive NF- $\mathrm{BB}$ activation observed in breast cancer patients. The impaired NF- $\kappa \mathrm{B}$ activation in primary mammary tumors of $M E p C-D K O / P y M T$ mice was accompanied by the induction of apoptotic cell death $(39,40)$, as evidenced by increased caspase-3 activity (Figure 2, C and D). Taken together, our results demonstrated that epsins play a critical role in mediating $\mathrm{NF}-\kappa \mathrm{B}$ activation in breast cancer development.

Despite the importance of epsins in NF- $\mathrm{KB}$ activation, how epsins modulate NF- $\mathrm{KB}$ activation in breast cancer remains entirely unknown. Given that TNFR1, the main receptor transducing the signals from TNF- $\alpha$, is one of the best-studied receptors in the canonical $\mathrm{NF}-\kappa \mathrm{B}$ pathway, we sought to elucidate the molecular mechanisms 
A
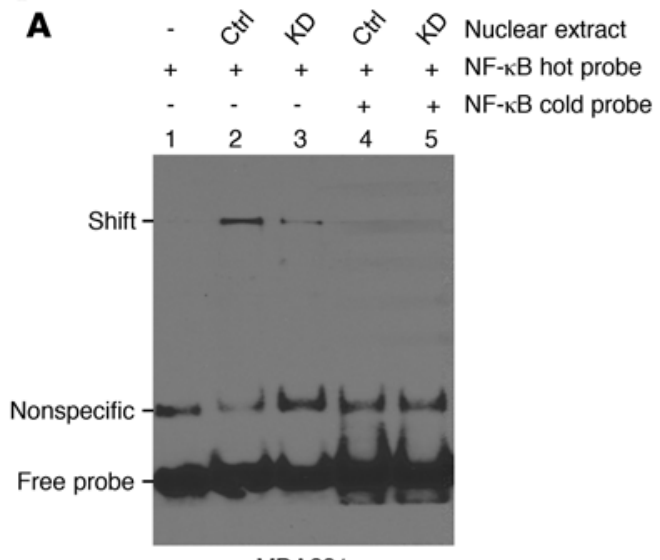

MDA231
B

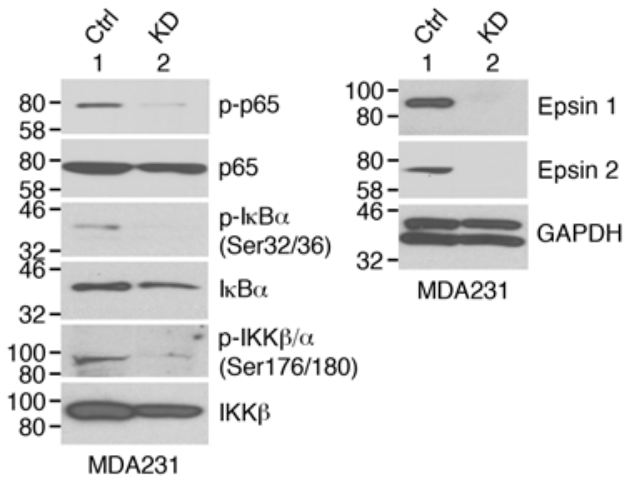

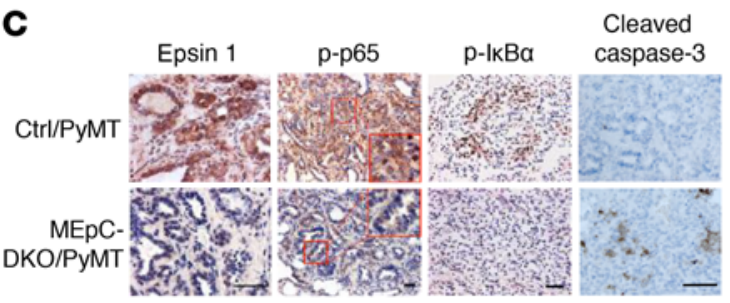

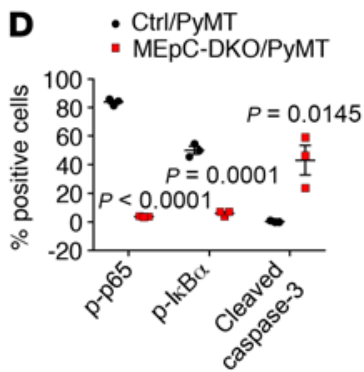

$\mathbf{E}$
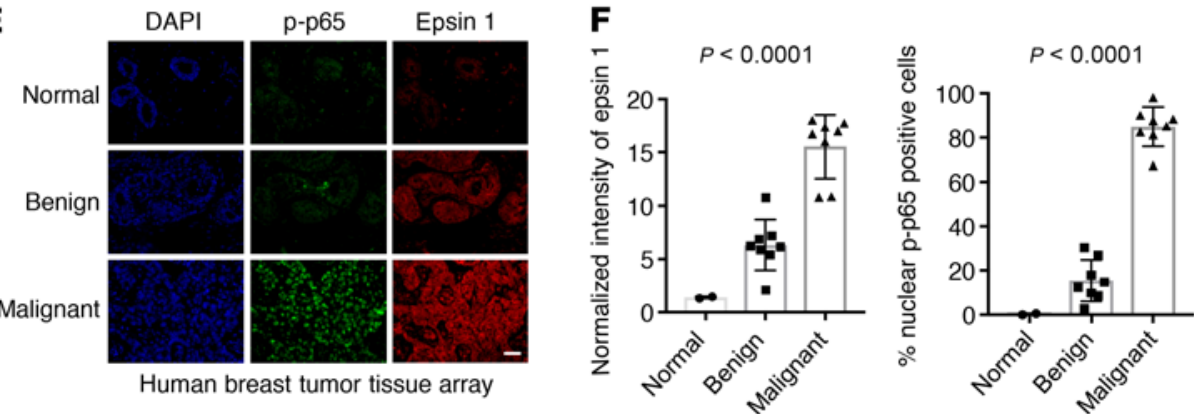

G
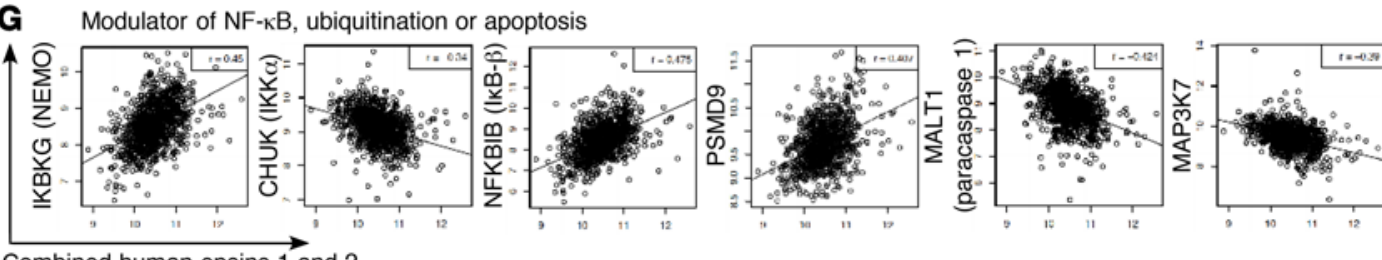

Combined human epsins 1 and 2

Figure 2. Epsin is a potentially novel regulator of NF-KB signaling in ER-negative breast cancer. (A) EMSA of NF- $\mathrm{kB}$ DNA binding activity ( $n=3$ independent experiments). Nuclear extracts ( $5 \mu \mathrm{g}$ ) were prepared from Ctrl and KD MDA231 cells and incubated with biotinylated probe (hot) or unlabeled as specific competitor (cold). Specific binding complex bands and free probes are indicated by arrows. (B) Western blot analyses of phosphorylated p65 ( $p$-p65), total p65, phosphorylated IKB- $\alpha$ (p-IKB- $\alpha$ ), total IKB- $\alpha$, phosphorylated IKK- $\beta / \alpha$ (p-IKK- $\beta / \alpha)$, and total IKK- $\beta$ in Ctrl and KD MDA231 cells ( $n=3$ independent experiments). (C and D) IHC analysis (C) and quantification (D) of epsin 1, p-p65, p-IKB- $\alpha$, and cleaved caspase-3 in primary tumors of Ctrl and MEpC-DKO MMTV-PyMT mice. A minimum of 3 randomly chosen fields ( $\times 10$ original magnification) of each tumor ( $n=3$ per genotype) were evaluated. Statistical values were calculated using unpaired, 2-tailed multiple $t$ tests. Data are presented as the mean \pm SEM. Scale bar: $100 \mu \mathrm{m}$. (E and $\mathbf{F})$ Immunofluorescence analysis (E) and quantification (F) of p-p65 and epsin 1 in human normal ( $n=2$ biologically independent samples), benign ( $n=8$ biologically independent samples), or malignant ( $n=8$ biologically independent samples) breast tissues. Statistical values were calculated using 1-way ANOVA followed by Tukey's multiple-comparisons test. Data are presented as the mean \pm SD. Scale bar: $50 \mu \mathrm{m}$. (C) The Cancer Genome Atlas Breast Cancer Carcinoma data collection (TCCA BRCA) GSEA at the $P$ value threshold of 0.01 unveiled positive correlation of NF- $\kappa B$-related genes with combined epsin $1 / 2$ expression. Gene expression data were converted to log ${ }_{2}$ scale. Notably, NEMO (IKBKC) is on top of the list. See also Supplemental Figure 2.

underlying epsin-mediated NF- $\kappa \mathrm{B}$ activation in response to TNF- $\alpha$. The prevailing model in the field is that the binding of TNFR1 by TNF- $\alpha$ induces a rapid and sequential recruitment of TNFR1-associated death domain protein (TRADD), receptor interacting protein 1 (RIP1), and others to TNFR1, forming the TNFR1 signaling complex (TNFRSC, complex I). Formation of this complex would, in turn, recruit IKK complex/signalosome (41). To determine whether epsin interacts with the TNFR1 complex upon TNF- $\alpha$ stimulation, we performed IP 
Table 1. NF-кB signatures and normalized enrichment scores (NES)

$\begin{array}{lccc}\text { NF-KB signatures } & \text { Genes } & \text { NES } & \begin{array}{c}\text { FDR } \boldsymbol{q} \\ \text { value }\end{array} \\ \text { TIAN_TNF_SIGNALING_VIA_NFKB } & 27 & 2.38 & <0.0001 \\ \text { HINATA_NFKB_TARGETS_FIBROBLAST_UP } & 73 & 2.37 & <0.0001 \\ \text { RASHI_NFKB1_TARGETS } & 19 & 2.34 & 0.0002 \\ \text { PHONG_TNF_TARGETS_UP } & 62 & 2.32 & 0.0004 \\ \text { HINATA_NFKB_TARGETS_KERATINOCYTES_UP } & 72 & 2.27 & 0.0007 \\ \text { ZHANG_RESPONSE_TO_IKK_INHIBITOR_AND_TNF_UP } & 182 & 2.24 & 0.0010 \\ \text { SANA_TNF_SIGNALING_UP } & 64 & 2.17 & 0.0018 \\ \text { MANTOVANI_NFKB_TARGETS_UP } & 30 & 2.06 & 0.0059 \\ \text { WANG_TNF_TARGETS } & 17 & 2.03 & 0.0076 \\ \text { DUTTA_APOPTOSIS_VIA_NFKB } & 31 & 2.00 & 0.0109 \\ \text { PHONG_TNF_RESPONSE_NOT_VIA_P38 } & 308 & 2.00 & 0.0112 \\ \text { CILMORE_CORE_NFKB_PATHWAY } & 12 & 1.98 & 0.0135 \\ \text { DEBOSSCHER_NFKB_TARGETS_REPRESSED_BY_GLUCOCORTICOIDS } & 11 & 1.88 & 0.0312 \\ \text { PID_NFKAPPABTYPICALPATHWAY } & 16 & 1.82 & 0.0478 \\ \text { SCHOEN_NFKB_SIGNALING } & 32 & 1.82 & 0.0480\end{array}$

Table 2. NF-кB signature gene list

\begin{tabular}{|ccc|}
\hline Gene name & Overlap signatures & Fold change \\
\hline CXCL3 & 6 & 30.2 \\
\hline IL8 & 8 & 27.9 \\
\hline CXCL1 & 6 & 20.1 \\
\hline IL1B & 5 & 15.4 \\
\hline COS2 & 3 & 11.0 \\
\hline CXCL2 & 6 & 6.2 \\
\hline BIRC3 & 7 & 4.8 \\
\hline TRAF1 & 6 & 3.9 \\
\hline NFKBIA & 12 & 3.7 \\
\hline IL6 & 6 & 3.6 \\
\hline TNFAIP3 & 9 & 3.6 \\
\hline CSF1 & 3 & 3.6 \\
\hline ETS1 & 3 & 3.0 \\
\hline CD83 & 6 & 2.7 \\
\hline MMP1 & 3 & 2.6 \\
\hline IER3 & 3 & 2.5 \\
\hline MMP9 & 3 & 2.4 \\
\hline ICAM1 & 7 & 2.3 \\
\hline PTX3 & 5 & 2.3 \\
\hline VECFA & 3 & 2.2 \\
\hline IL32 & 3 & 2.2 \\
\hline
\end{tabular}

with an anti-TNFR1 antibody using mouse primary fibroblastic cells. In response to TNF- $\alpha$ stimulation, IP of TNFR1 pulled down TRADD, RIP1, HOIP, and NEMO, as expected (Figure 3A). Interestingly, we found that epsin was also recruited to the TNFR1 complex (Figure 3A). To the best of our knowledge, this is the first observation that epsin is a potentially novel native component involved in TNFR1 signaling.

Given that epsins function as endocytic adaptor proteins, it is plausible that epsins modulate TNF- $\alpha$-stimulated NF- $\mathrm{KB}$ signaling transduction by controlling endocytosis of TNFR1. TNF- $\alpha$-induced
TNFR1 internalization was analyzed by confocal microscopic visualization of fluorescently labeled TNF- $\alpha$ bound to the receptor in control and epsin-deficient primary fibroblasts (Supplemental Figure 3A). Regardless of the presence or absence of epsins, 5 minutes of TNF- $\alpha$ stimulation induced TNFR1 internalization and colocalization with epsin 1 , and 15 minutes of TNF- $\alpha$ stimulation promoted TNFR1 colocalization with the lysosomal marker LAMP1, suggesting that TNF- $\alpha-$ induced internalization of TNFR1 occurs independent of epsins. To confirm this finding, a cell surface biotinylation experiment was performed, and surface TNFR1 levels in control and epsin-deficient MDA231 cells after TNF- $\alpha$ stimulation were measured by streptavidin bead pulldown followed by Western blot analysis for TNFR1 (Supplemental Figure 3B). Equivalent reductions in surface TNFR1 levels after 2 minutes, relative to 0 -minute $\mathrm{TNF}-\alpha$ stimulation, were observed in both control and epsin-deficient cells, suggesting that TNFR1 internalization occurred normally in the absence of epsins. Collectively, these data suggest that epsins are not required for TNFR1 endocytosis but are obligatory for NF- $\mathrm{BB}$ activation. Given that epsins control Notch signaling, which also promotes the tumor-initiating cell phenotype in breast cancer (42), we overexpressed an active form of Notch, the Notch intracellular domain (NICD), in epsin-deficient MDA231 cells to restore Notch signaling. We uncovered via Western blotting that restoring Notch signaling did not rescue impaired NF- $\kappa B$ activation (data not shown), suggesting that epsins regulate $\mathrm{NF}-\kappa \mathrm{B}$ signaling independently of Notch activity.

The ubiquitin system is crucial for NF- $\mathrm{BB}$ regulation in terms of mediating the formation and stabilization of the TNFR1 complex (43). Through conjugation of different types of polyubiquitin chains, mainly K63 ubiquitin and/or linear ubiquitin chains, the function of the components of the TNFR1 complex is fine-tuned in a timely and selective manner (44). Given that epsins were recruited to the TNFR1 complex in response to TNF- $\alpha$ stimulus (Figure $3 \mathrm{~A}$ ), and the ubiquitin-interacting motif (UIM) of epsin proteins is responsible for their interaction with ubiquitinated proteins $(45$, 46), including components of the TNFR1 complex, we sought to determine whether the epsin UIM was critical for the interaction of epsins with the TNFR1 complex. To this end, we coexpressed HA-tagged full-length epsin 1 or UIM-deficient epsin 1 (epsin 1 $\triangle$ UIM) with either Flag-tagged WT RIP1 or mutant RIP1 (K377R) in 293T cells and performed IP using anti-HA antibodies. Our results revealed that in response to TNF- $\alpha$ treatment, full-length epsin 1 but not epsin $1 \Delta$ UIM coimmunoprecipitated RIP1, and K377R mutation, which disrupts the functional linear ubiquitination site in $\operatorname{RIP} 1(47,48)$, reduced the binding of RIP1 with epsin 1 (Figure 3B and Supplemental Figure 3C), suggesting that epsin binds to RIP1 in a UIM-dependent manner, in part by interacting with linear ubiquitin chains conjugated to RIP1. Emerging studies have unveiled the importance of linear ubiquitination in NF- $\mathrm{BB}$ activation (14, 49-51). Accordingly, we found that the epsin UIM preferentially bound linear polyubiquitin chains relative to K63- or K48linked polyubiquitin chains (Figure $3 \mathrm{C}$ and Supplemental Figure 3D). To date, the only known E3 ligase that specifically catalyzes 
A

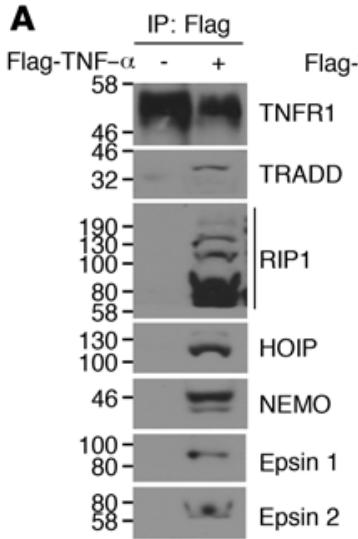

Lysates

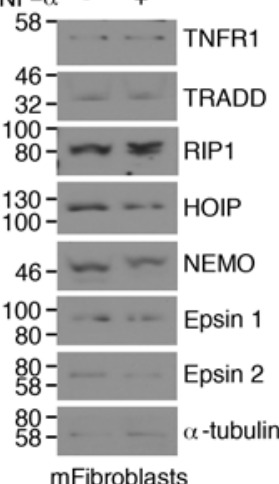

D
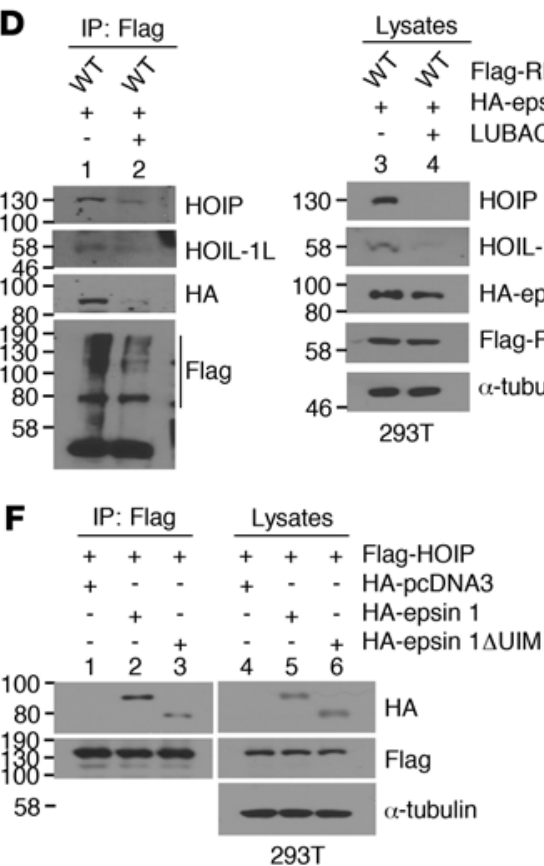

B

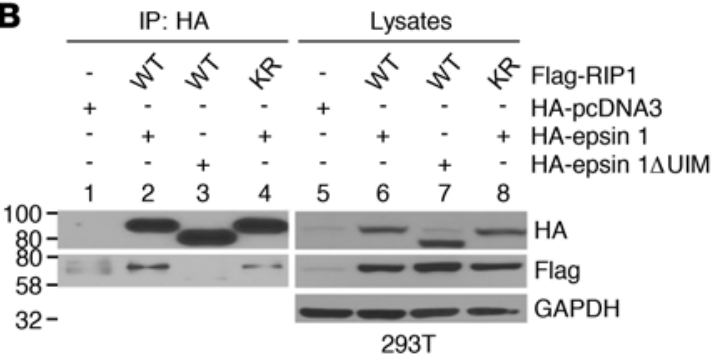

C

Pull-down

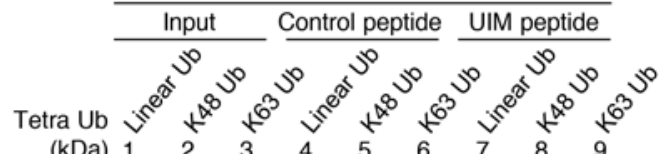

$\begin{array}{llllllllll}(\mathrm{kDa}) & 1 & 2 & 3 & 4 & 5 & 6 & 7 & 8 & 9\end{array}$

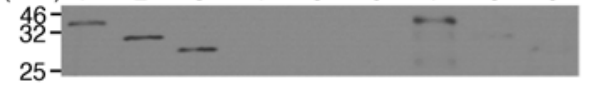

E
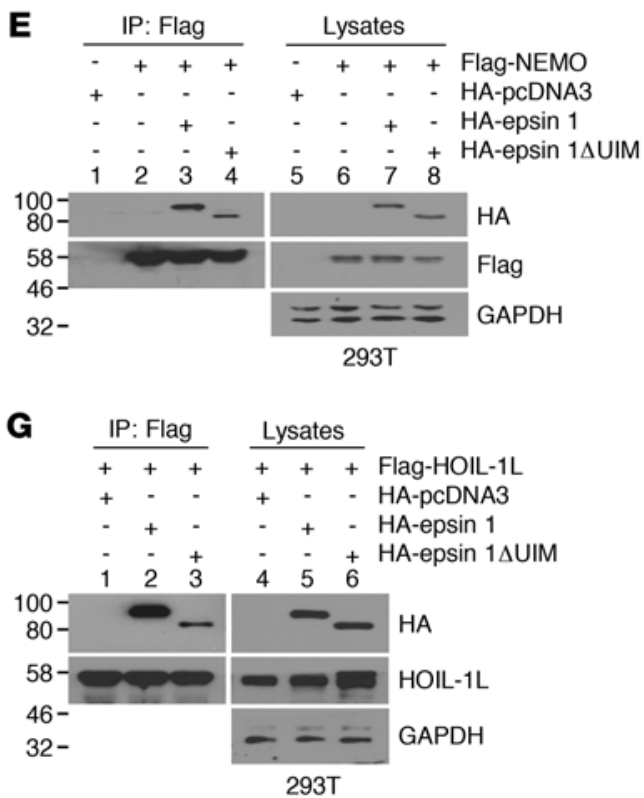

Figure 3. Recruitment of epsin to TNFR1 complex in response to TNF- $\alpha$ stimulus and epsin interacts with LUBAC and its substrates in a UIM-dependent manner. (A) Mouse primary fibroblasts (mFibroblasts) were stimulated with Flag-TNF- $\alpha(1 \mu \mathrm{g} / \mathrm{mL})$ for 5 minutes, lysed, and pulled down using anti-Flag $\mathrm{M} 2$ affinity gel at $4^{\circ} \mathrm{C}$ for 16 hours, following by Western blot analysis of epsin, NEMO, and components of TNF receptor signaling complex (TNF-RSC). (B) Western blot analysis of anti-HA IP in 293T cells cotransfected with Flag-tagged RIP1 (or K337R mutant RIP1) and HA-epsin 1 or HA-epsin $1 \Delta$ UIM after TNF- $\alpha$ stimulation $(20 \mathrm{ng} / \mathrm{mL}$ ) for 5 minutes. (C) Western blot analysis of linear K48-linked or K63-linked tetraubiquitin (tetraUb) pulled down by biotinylated scramble control peptide or epsin UIM peptide. (D) Western blot analysis of anti-Flag IP in Ctrl (generated by transfecting scrambled siRNA using lipofectamine RNAiMAX) or LUBAC KD (generated by transfecting siRNA against HOIP and HOIL-1L, respectively) 293T cells, which were cotransfected with Flag-WT RIP1 and HA-epsin 1 after TNF- $\alpha$ stimulation $(20 \mathrm{ng} / \mathrm{mL})$ for 5 minutes. (E-G) Western blot analysis of anti-Flag IP in $293 T$ cells cotransfected with Flag-NEMO (E), Flag-HOIP (F), or Flag-HOIL-1L (C), and HA-epsin 1 or HA-epsin 1 UUIM after TNF- $\alpha$ stimulation (20 ng/mL) for 5 minutes. Results are representative of 3 independent experiments (A-G). See also Supplemental Figure 3.

linear ubiquitination is the LUBAC, which is composed of HOIP, the central catalytic unit of LUBAC, HOIL-1L, and/or SHARPIN. We found that in response to TNF- $\alpha$ treatment, the recruitment of HA-tagged full-length epsin 1 to Flag-tagged WT RIP1 was largely abolished in HOIP and HOIL-1L double knockdown 293T cells as compared with controls (Figure 3D and Supplemental Figure 3E). Our co-IP result recapitulated the loss of interaction between epsin 1 and RIP1 mutants, as described above. Next, given that NEMO has a high preference for linear ubiquitination in that it displays a 100-fold greater affinity for linear ubiquitin than K63 ubiquitin (11, $14,50,52)$, we tested whether epsin 1 also interacts with NEMO by performing co-IP in 293T cells cotransfected with vectors expressing Flag-tagged NEMO and HA-tagged full-length epsin 1 or epsin $1 \Delta$ UIM. Likewise, we observed that NEMO coprecipitated with full-length epsin 1 but UIM deficiency decreased the binding of epsin 1 with NEMO upon TNF- $\alpha$ stimulation (Figure 3E and Supplemental Figure 3F), suggesting that epsin 1 interacts with NEMO, partially relying on its UIM. Given that RIP1 and NEMO are among several bona fide substrates for linear ubiquitination $(49,53)$ and epsin 1 interacts with both RIP1 and NEMO, we hypothesized that epsins may directly interact with LUBAC. Indeed, in 293T cells expressing HA-tagged full-length epsin 1 or epsin $1 \Delta$ UIM with 

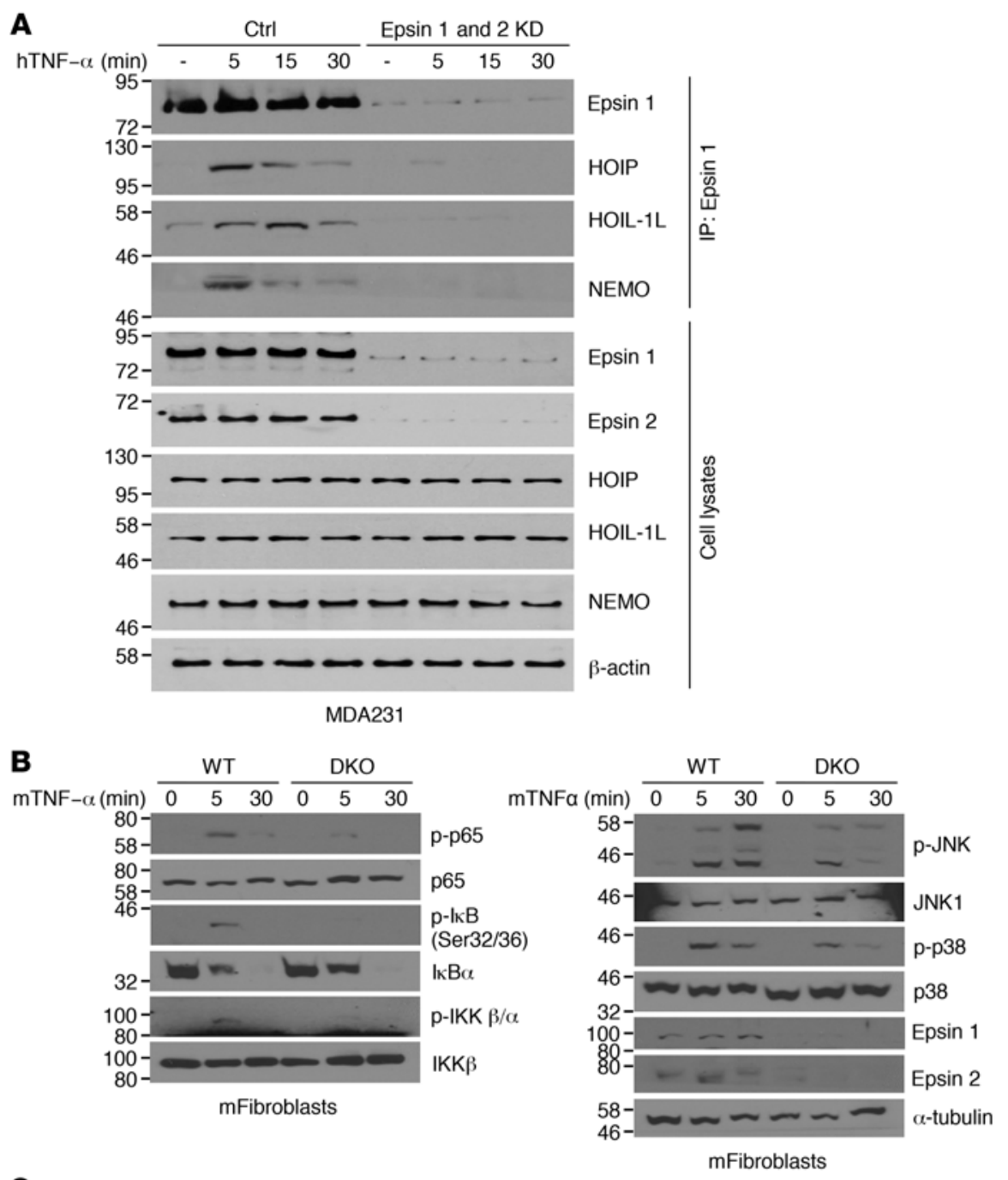

C
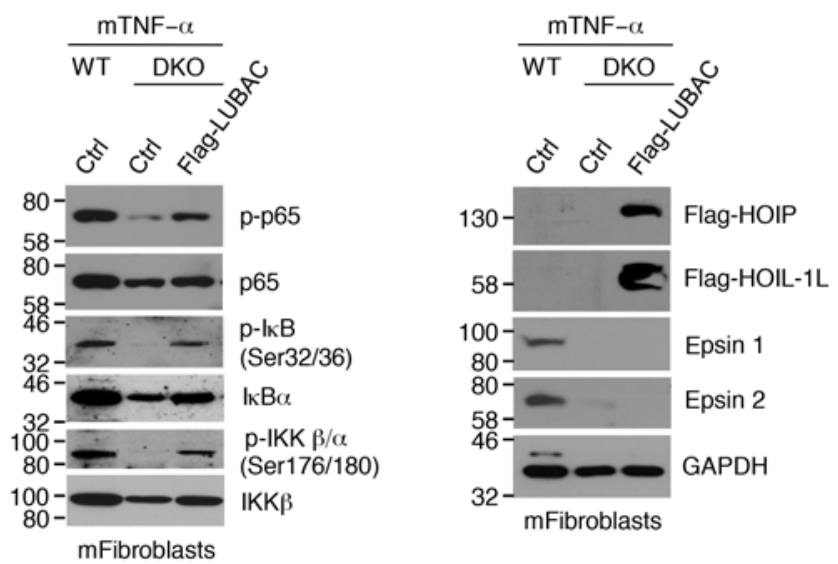

Figure 4. The interaction of epsin with LUBAC is critical for NF-kB activation. (A) Ctrl MDA231 cells (generated by transfecting scrambled siRNAs using lipofectamine RNAiMAX) or epsin 1 and 2 KD MDA231 cells (generated by transfecting siRNAs against epsin 1 and epsin 2, respectively) were stimulated with human TNF- $\alpha$ (hTNF- $\alpha ; 50 \mathrm{ng} / \mathrm{mL}$ ) for the indicated time, lysed, and pulled down with epsin 1 antibody at $4^{\circ} \mathrm{C}$ for 4 hours, followed by Western blot analysis of epsin $1, \mathrm{HOIP}, \mathrm{HOIL}-1 \mathrm{~L}$, and NEMO. (B) Western blot analysis of mouse TNF- $\alpha$-induced (mTNF- $\alpha$-induced) phosphorylation of p65, IKB- $\alpha$, IKK- $\beta / \alpha$, JNK, and p38 in WT and DKO mouse primary fibroblasts. Both groups were treated with $50 \mathrm{ng} / \mathrm{mL}$ mTNF- $\alpha$ for the indicated time. (C) Western blot analysis of mTNF- $\alpha$-induced ( $50 \mathrm{ng} /$ $\mathrm{mL}$, final concentration, 5 minutes) phosphorylation of $\mathrm{p} 65$, IKB- $\alpha$, or IKK- $\beta$ in WT and DKO mouse primary fibroblasts, with or without overexpression of Flag-tagged HOIP and Flag-tagged HOIL-1L. Results are representative of 3 independent experiments (A-C). either Flag-tagged HOIP or HOI-1L, co-IP experiments revealed that LUBAC (including both HOIP and HOIL-1L) strongly bound epsin 1 in response to TNF- $\alpha$ stimulus, and the absence of UIM attenuated binding (Figure 3, $\mathrm{F}$ and $\mathrm{G}$, and Supplemental Figure 3, G and H). To further delineate the endogenous interaction of epsin with LUBAC, MDA231 cells were treated with TNF- $\alpha$ and cell lysates were subjected to anti-epsin IP in the presence or absence of epsins (Figure 4A). TNF- $\alpha$ stimulation promoted epsin 1 coprecipitation with LUBAC and NEMO, whereas epsin deficiency abolished the presence of LUBAC and NEMO in anti-epsin 1 immunoprecipitates (Figure 4A and Supplemental Figure 3I). Additionally, knockdown of LUBAC reduced recruitment of epsins to the TNFR1 complex (Supplemental Figure 3J). In LUBAC$\mathrm{KO}$ mice and cells derived from these mice, NF- $\mathrm{KB}$ signaling is suppressed (49-51). Similarly, deficiency of epsins 1 and 2 in mouse primary fibroblasts stimulated with TNF- $\alpha$ resulted in impaired activation of the NF- $\mathrm{KB}$ pathway, including diminished phosphorylation of IKK, IKB- $\alpha$, and p65 (Figure 4, B and C, and Supplemental Figure $3 \mathrm{~K})$ compared with the controls. Conversely, overexpressing LUBAC in epsindeficient cells rescued the NF- $\mathrm{\kappa B}$ activation defect (Figure $4 \mathrm{C}$ and Supplemental Figure $3 \mathrm{~K})$, suggesting that the interaction of epsin with LUBAC is critical for NF-kB activation.

Despite the finding that epsin was associated with multiple ubiquitinated components in the TNFR1 complex, NEMO gene expression correlated particularly strongly with epsin 1 and 2 expression in human breast cancer (Figure 2G). We sought to examine in higher resolution the interaction of epsins and NEMO in breast cancer. IP of TNFR1 in epsin-deficient MD231 cells efficiently pulled down TRADD and RIP1; however, coprecipitation of NEMO with TNFR1 was dramatically decreased owing to epsin loss (Supplemental Figure $4 \mathrm{~A})$, suggesting that epsin may directly interact with NEMO, and the epsin-NEMO interaction plays an imperative role when epsins potentiate NF- $\mathrm{\kappa B}$ activation.

To test the hypothesis that epsin binds NEMO directly, we decided to determine which domain(s) in epsin are responsible for NEMO binding. Full-length epsin 1 or truncation epsin mutants comprising the UIM, N-terminal homolog (ENTH), or DPW-NPF 
A ENTH UIM DPW NPF

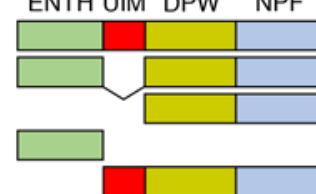

Epsin 1 Epsin $1 \Delta$ UIM DPW-NPF ENTH $\triangle \mathrm{ENTH}$

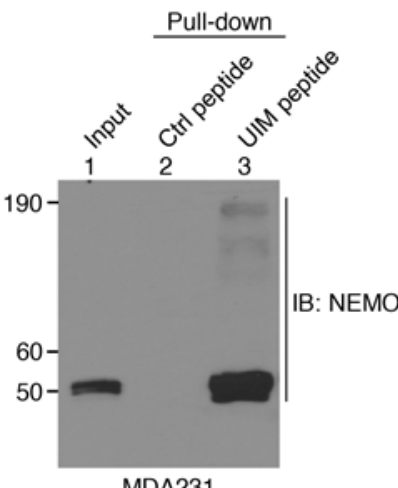

MDA231

C
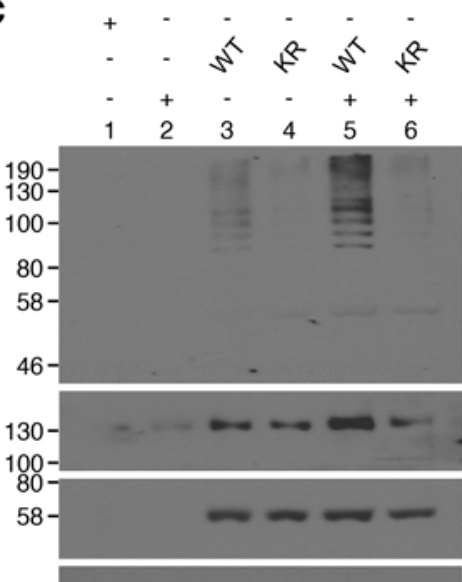

Empty vector

Flag-NEMO

Xpress-epsin 1

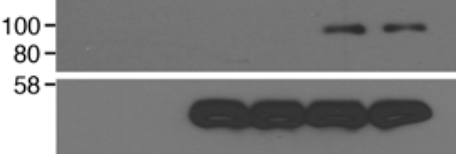

Xpress-epsin 1

Linear

ubiquitin

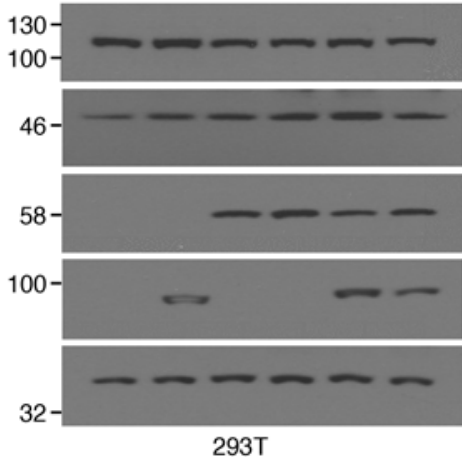

Flag-NEMO

HOIP

HOIL-1L

Flag-NEMO

Xpress-epsin 1

GAPDH

$293 T$

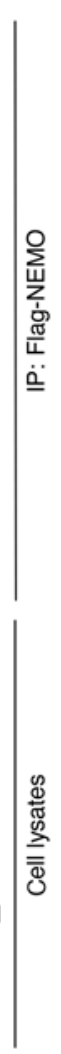

Input Pull-down

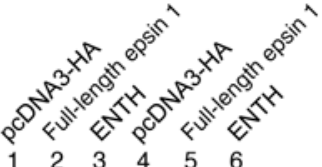

Input $\longrightarrow$ Pull-down

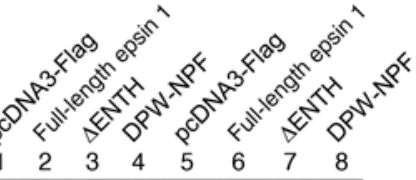

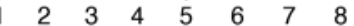

HA

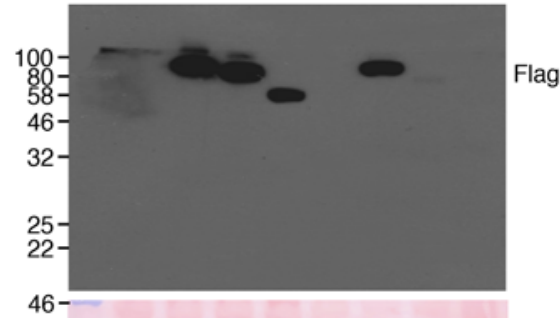

NEMO 32

NEMO

D

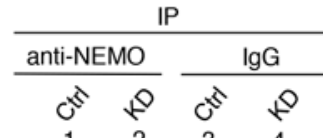
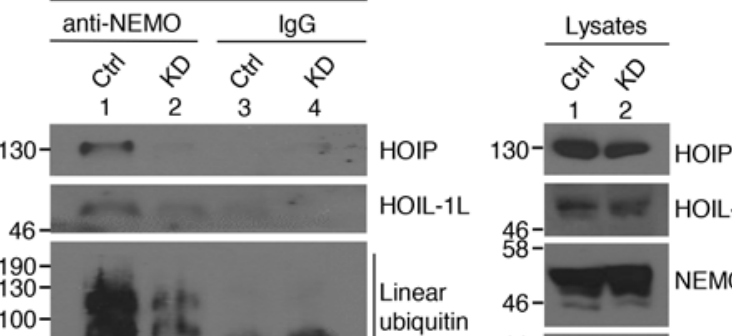

$80-$

58
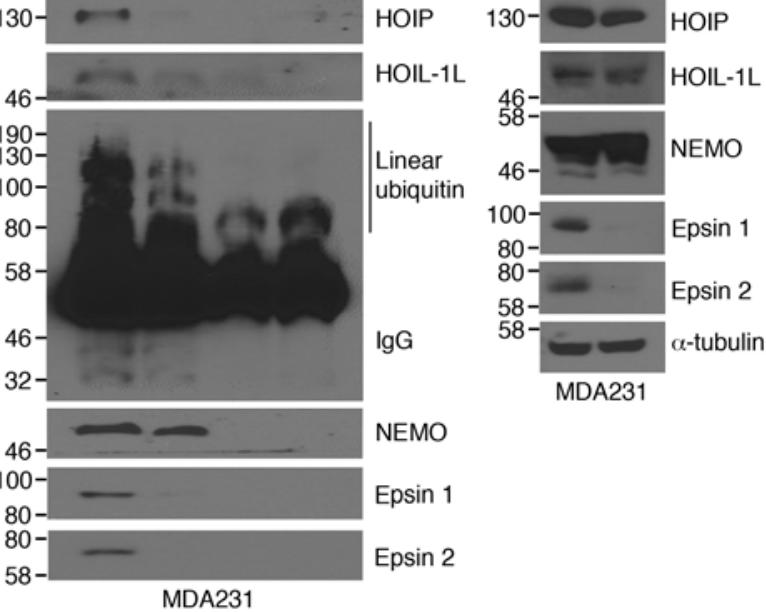

$\alpha$-tubulin

MDA231
E

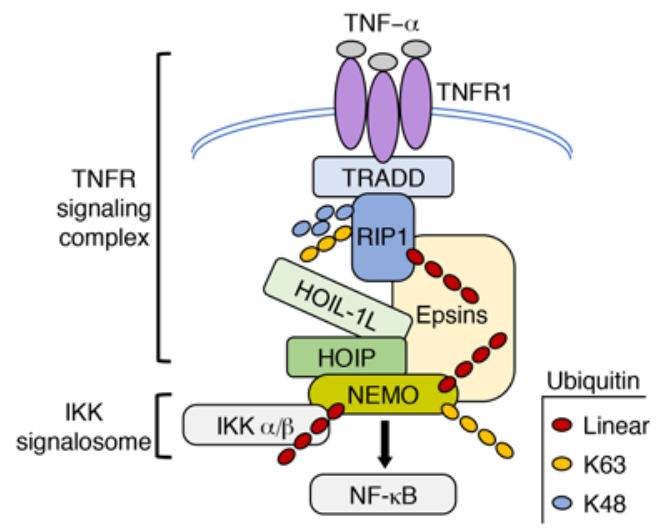

Figure 5. UIM and ENTH domains account for binding of epsin to NEMO, and epsin promotes NEMO ubiquitination by facilitating its association with LUBAC. (A) Western blot analysis of NEMO pulled down by biotinylated scramble control peptide or epsin UIM peptide from MDA231 cell lysate. (B) Pulldown assay of full-length epsin 1, ENTH domain of epsin 1, ENTH domain-deficient epsin 1, and DPW-NPF domains of epsin 1 by purified NEMO. (C) Western blot analysis of anti-Flag IP in 293T cells cotransfected with Flag-NEMO (WT) or Flag-NEMO K285/309R mutant (KR) without or with Xpress-epsin 1 after TNF- $\alpha$ stimulation $(20 \mathrm{ng} / \mathrm{mL}$ ) for 5 minutes. (D) Western blot analysis of NEMO ubiquitination by anti-NEMO IP in Ctrl and KD MDA231 cells. (E) Proposed working model illustrating an epsin-dependent increase in NEMO polyubiquitination and assembly of TNFR1 complex in breast cancer. Results are representative of 3 independent experiments (A-D). See also Supplemental Figure 4. 
domains were generated and used for a pulldown assay (Figure 5A). A biotinylated epsin UIM peptide efficiently pulled down nonubiquitinated and ubiquitinated NEMO from MDA231 cell lysates relative to a scrambled control peptide (Figure 5A and Supplemental Figure 4B), suggesting that the epsin UIM is capable of interacting with NEMO regardless of its ubiquitination state, which is consistent with our previously reported epsin UIM-VEGFR2 interaction (37). Successful pulldown of full-length epsin by purified NEMO protein produced in bacteria further supported the hypothesis that the epsin-NEMO interaction can occur independently of NEMO ubiquitination (Figure 5B and Supplemental Figure 4C). In searching for other regions in epsin responsible for the epsin-NEMO interaction, we found that the ENTH domain (Figure 5C and Supplemental Figure 5C), but not the DPW-NPF domain (Figure 5D and Supplemental Figure 4C), interacted with purified NEMO protein, suggesting that epsins physically bind NEMO through a direct protein-protein interaction. Although both epsin UIM and ENTH were involved in the epsin-NEMO interaction, deletion of the UIM greatly reduced binding of epsin to NEMO (Figure 3E), reflecting a dominant role of epsin UIM in mediating the epsin-NEMO interaction. Taken together, we have uncovered a potentially novel molecular mechanism underlying the bona fide epsin-NEMO interaction that is both dependent and independent of NEMO ubiquitination (Figure 3, E and H, and Figure 5, A-D).

Linear ubiquitination chains on NEMO induce a conformational change of NEMO that is thought to facilitate IKK complex assembly and IKK- $\beta$ activation (16-19). We hypothesize that epsins promote NEMO linear polyubiquitination by facilitating the binding of E3 ligase-containing LUBAC to NEMO. Because lysine 285 (K285) and lysine 309 (K309) in NEMO are linear ubiquitination sites that can be ubiquitinated by LUBAC $(11,14,54)$, we mutated K285 and K309 to arginine in a Flag-tagged NEMO construct, cotransfected WT NEMO or NEMO K285R/309R double mutant (NEMO KR), without or with Xpress-tagged epsin 1 (21) in 293T cells, and then performed co-IP analysis. The results demonstrated that in the presence of exogenously expressed epsin 1 , binding of HOIP to NEMO was dramatically augmented, with a parallel elevation of NEMO linear ubiquitination (probed by a linear ubiquitinspecific antibody; Figure 5C, lane 5, and Supplemental Figure 4D), relative to endogenous epsin (Figure 5C, lane 3, and Supplemental Figure 4D). Intriguingly, mutation of K285 and K309 strikingly reversed the increase in binding of HOIP to NEMO to an extent that was similar to endogenous epsin despite the presence of exogenously expressed epsin (Figure 5C, lanes 4 and 6, and Supplemental Figure 4D). These results provide compelling evidence that epsins play a crucial role in promoting the binding of HOIP to NEMO and increasing its linear ubiquitination when epsins are highly expressed, a situation often existent in breast cancer cells. Furthermore, the presence of intact K285 and K309 linear ubiquitination sites was imperative for enhanced binding of HOIP to NEMO, which was mediated by epsin. Interestingly, binding of HOIP and HOIL-1L to NEMO was minimally affected by mutation of K285 and K309 in the presence of endogenous or low levels of epsin (Figure 5C, lanes 3 and 4, and Supplemental Figure 4D), suggesting that epsin was dispensable for initial recruiting of HOIP and HOIL-1L to NEMO and for maintaining the normal level of $\mathrm{NF}-\kappa \mathrm{B}$ activity in a healthy condition. Consistent with this finding, epsin deficiency markedly reduced the association of NEMO with HOIP, the catalytic subunit of LUBAC, thus decreasing NEMO linear ubiquitination, in ER-negative breast cancer MDA231 cells (Figure 5D and Supplemental Figure 4E).

Next, we wanted to investigate the functional consequences of the epsin-NEMO interaction. We hypothesized that constitutive activation of $N F-\kappa B$ would restore impaired NF- $\kappa$ B signaling due to epsin deficiency, thus promoting breast cancer development in vivo. We crossed MEPC-DKO MMTV-PyMT mice with mice harboring a transgene encoding a constitutively active IKK2 (IKK2ca mice) (Supplemental Figure 5A), which is a well-established model to induce constitutive activation of NF- $\mathrm{B}$ (55). Notably, IKK2 (also called IKK- $\beta$ ) expression was elevated in breast tumors as revealed by Western blotting of multiple tissue homogenates isolated from $M E p C-D K O / I K K 2 \mathrm{ca}^{f /+}$ mice (data not shown). This mimicked a promotion of IKK activation. Levels of p-p65 and p-IкB- $\alpha$ in tumor tissues of $M E p C-D K O / I K K 2 c a^{f /+}$ mice were significantly restored compared with control mice, suggesting that expression of constitutively active IKK2 activates NF- $\mathrm{KB}$ signaling and compensates for epsin loss in $M E p C-D K O / I K K 2 c a^{f /+}$ mice (Supplemental Figure 5B). Moreover, IKK2ca expression rescued the tumor-resistant phenotype of MEpC-DKO mice reflected by accelerated tumor growth (Figure 6A). To substantiate our findings, a previously reported potent NF- $\mathrm{BB}$ inhibitory NEMO binding domain (NBD) peptide $(56,57)$ was i.p. injected into $M M T V-P y M T$ tumor mice, mimicking a decrease in IKK recruitment, and was found to potently block early tumorigenesis, as revealed by whole-mount staining of mammary glands (Figure 6B). This provided further support for our observation that impaired NF- $\kappa \mathrm{B}$ signaling impeded breast tumorigenesis. Furthermore, expressing epsin 1 but not epsin $1 \Delta \mathrm{UIM}$ in epsin-deficient MDA231 cells restored NF- $\mathrm{B}$ signaling (Figure 6C and Supplemental Figure 5C). Additionally, relative to a scrambled control peptide, a chimeric epsin UIM peptide designed to competitively impair endogenous epsin function (36) effectively inhibited phosphorylation of p65 in MDA231 cells and reduced tumor development in MMTV-PyMT mice as well as in a triple-negative breast cancer patient-derived xenograft (PDX) model $(58,59)$, in part by suppressing NF- $\mathrm{B}$ activation (Figure $5, D^{-} \mathrm{G}$ and Supplemental Figure 5, D-F). This suggests a unique opportunity to inhibit tumor-intrinsic NF- $\mathrm{KB}$ signaling by targeting the UIM, which represents a potentially new strategy for therapeutic intervention of ER-negative breast cancer.

\section{Discussion}

Our detailed mechanistic analyses revealed that the epsin UIM was indispensable for the interaction between epsin and NEMO (Figure 3, E and H, and Figure 4, A-D), which preferentially bound linear ubiquitin chains often found in the components in the TNFR1 complex, including RIP1. Simultaneous binding of epsin to LUBAC and NEMO (via the epsin ENTH domain) promoted NEMO linear ubiquitination by LUBAC, which in turn facilitated the binding of the epsin UIM to linear ubiquitinated NEMO. In this scenario, because of the heightened expression of epsins in breast cancer, abundant NEMO linear polyubiquitin within the TNFR1 complex, which possibly leads to more IKK recruitment and/or promotes further IKK activation (15), supports the concept that the epsin-NEMO interaction sustains NF- $\mathrm{KB}$ activation in breast 
A
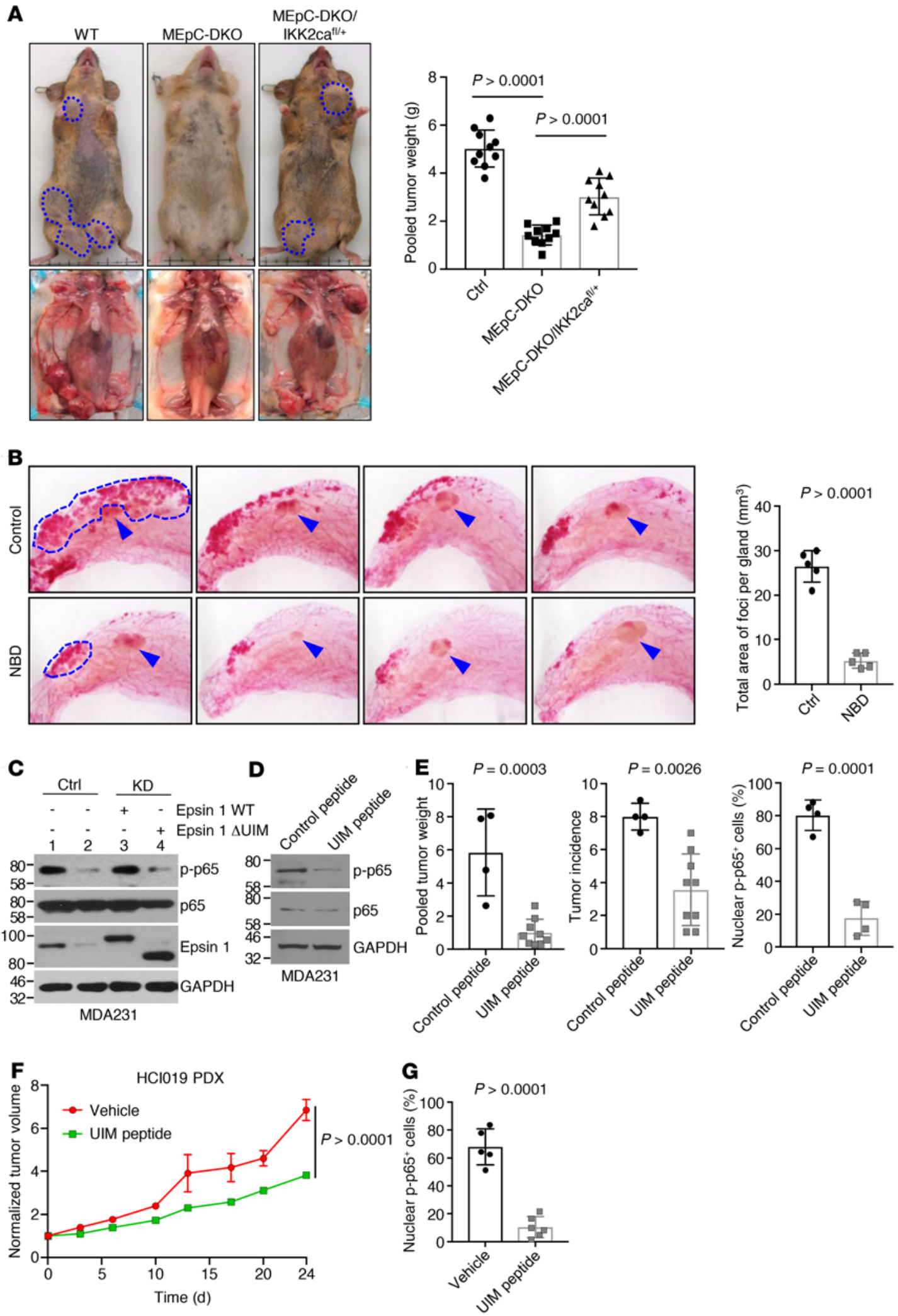
Figure 6. Epsin-mediated NF-кB activation in breast cancer development. (A) Analysis of mammary tumors in Ctrl, MEpC-DKO, or MEpC-DKO/IKK2 $\mathrm{ca}^{\mathrm{fl} /+}$ MMTV-PyMT mice ( $n=10$ each). Statistics: 1-way ANOVA and Tukey's multiple-comparisons test (mean $\pm \mathrm{SD}$ ). (B) Whole-mount staining and quantification of gland lesions in MMTV-PyMT mice treated with Ctrl or NBD peptides $(2 \mathrm{mg} / \mathrm{mL})(n=5$ biological replicates). MMTV-PyMT mice were i.v. injected (100 $\mu \mathrm{L}$ per mouse) with peptides for 2 weeks and euthanized at 8 weeks. Statistics: unpaired, 2-tailed $t$ test (mean \pm SD). (C) Western blots of constitutive p65 phosphorylation in Ctrl or KD MDA231 cells transfected with epsin 1 (KD + epsin 1WT) or epsin $1 \Delta \mathrm{UIM}$ (KD + epsin $1 \Delta \mathrm{UIM}$ ) vectors ( $n=3$ independent experiments). (D) Western blots of p-p65, p65, and GAPDH in MDA231 cells treated with $10 \mu \mathrm{M}$ scramble control or epsin UIM peptides for 15 hours ( $n=3$ independent experiments). (E) Analysis of primary tumors (weight, incidence, p-p65 staining) from MMTV-PyMT mice treated with Ctrl or epsin UIM peptide ( $n=4$ Ctrl peptide-treated mice and 9 UIM peptide-treated mice). MMTV-PyMT mice were i.p. injected with Ctrl or epsin UIM peptide $(10 \mathrm{mg} / \mathrm{kg}$, $100 \mu \mathrm{L}$ each) and were given 3 i.p. injections per week for 8 weeks. Statistics: unpaired, 2-tailed $t$ test (mean \pm SD). (F) Analysis of tumor volume in shamor epsin UIM peptide-treated triple-negative breast cancer PDX mice $(n=5$ sham-treated mice and 6 UIM peptide-treated mice). When tumor volume reached $150-250 \mathrm{~mm}^{3}$, PDX-bearing NSC mice were i.v. injected with peptides $(40 \mathrm{mg} / \mathrm{kg}, 100 \mu \mathrm{L}$ each) and were subsequently given 3 i.v. injections per week. Statistics: 2-way ANOVA and Sidak's multiple-comparisons test (mean \pm SEM). (G) Quantification of $\mathrm{p}-\mathrm{p} 65 \mathrm{IHC}$ in primary tumors from PDX mice (n = 5 sham-treated mice and 6 UIM peptide-treated mice). Statistics: unpaired, 2-tailed $t$ test (mean \pm SD). See also Supplemental Figure 5 .

cancer (Figure 4E). It is also thought that NEMO binding to linear ubiquitin chains induces a conformation change that is required to activate the IKK complex. After linear ubiquitin chains interact with the NEMO UBAN region, conformational changes occur within this region, and these changes affect the interaction of the NEMO N-terminal region with IKKs and regulate the activity of the IKK complex (15-19). By interacting with LUBAC, the presence of epsin in the TNFR1 complex would conceivably enhance the linear ubiquitination of components of the TNFR1 complex by LUBAC, which would likely lead to further augmentation of IKK activity and result in NF- $\kappa \mathrm{B}$ activation. Intriguingly, epsin itself is frequently modified by monoubiquitination in a UIM-dependent manner, which presumably facilitates assembly of a complex that is highly modified by ubiquitination around epsin, given that the epsin UIM serves as an attractive docking site for E3 ligases to conjugate ubiquitin onto their target proteins $(45,46)$. Despite the fact that epsin itself would be one of the mounting substrates for LUBAC, whether the scaffolding function or the catalytic activity of HOIP (i.e., the presence of linear chains as a platform for the recruitment of epsins) is needed in this process warrants further investigation. Our data support the idea that epsin may function as irreplaceable machinery in facilitating LUBAC to attach linear ubiquitin chains to NEMO and retain NEMO in the TNFR1 complex, establishing a positive feedback loop that drives IKK activation and maintains a high level of NF- $\kappa \mathrm{B}$ activity in breast cancer.

A decade ago, ER-negative breast cancer, a more aggressive and detrimental subtype, was demonstrated to have elevated and activated NF- $\mathrm{B}(1,2,5,6)$. Here, we describe potentially novel molecular mechanisms underlying the heightened NF- $\kappa \mathrm{B}$ activity in ER-negative breast cancer. Epsins, the family of ubiquitinbinding endocytic adaptors, tie to the linear ubiquitin network within the TNFR1 complex by interacting with the linear ubiquitinating enzyme, LUBAC, where epsin binds its bona fide partner NEMO via its ENTH domain, promotes NEMO linear ubiquitination by LUBAC, and provides scaffolds for recruiting other components of the IKK complex to the TNFR1 complex. This results in heightened IKK activation and elevated NF- $\mathrm{KB}$ signaling essential for the development of ER-negative breast cancer. Our studies offer a concrete molecular basis for developing inhibitory strategies that target epsin as a potentially novel ER-negative breast cancer treatment. We anticipate that future translational research studies may yield important information on whether targeting epsins may be a viable strategy for personalized anticancer treatments.

\section{Methods}

Mice. We report a strategy to generate an Epn1 tissue-specific and Epn2 global KO mouse model $(24,28)$. To create a mammary epithelial cellspecific KO of Epn1 and a global KO of Epn2 mouse model, we crossed $E p n 1^{f l / f l} E p n 2^{-/-}$mice with $M M T V$-Cre transgenic mice purchased from the Jackson Laboratory (stock 003553), which express Cre recombinase specifically in the mammary epithelial cells (60), and generated mammary epithelium-specific double KO mice (MEpC-DKO). These mice were then backcrossed onto the C57BL/6 background. To generate the epithelial cell-specific epsin 1 and 2 double KO spontaneous breast cancer mouse model, we crossed $M E p C$-DKO mice with $M M T V$ PyMT transgenic mice (purchased from the Jackson Laboratory, stock 002374). Given that MMTV-PyMT mice are on the FVB genetic background, we backcrossed MMTV-PyMT mice onto the C57BL/6 background. We then bred the resultant mice with (a) WT C57BL/6 mice to obtain WT/PyMT mice, and (b) MEpC-DKO mice to obtain Epn $1^{f / f / f}$ Epn2 $2^{-/}$MMTV-PyMT MMTV-Cre mice (MEpC-DKO/PyMT mice on the $\mathrm{C} 57 \mathrm{BL} / 6$ background). Given that epsins 1 and 2 play a redundant role based on our previous study, for additional controls, we utilized $E^{2} n^{+/+}$Epn $2^{-/-}$MMTV-PyMT MMTV-Cre and Epn1 fl/fl Epn2 $2^{-/-}$MMTVPyMT littermates. The control mice cohort (Ctrl/PyMT) for MEpCDKO/PyMT, consisting of both Epn1//+ Epn2-/- MMTV-PyMT MMTVCre with a single copy of MMTV-Cre, and Epn $1^{f / f l}$ Epn $2^{-/-} M M T V-P y M T$ littermate controls lacking the single copy of $M M T V$-Cre, exhibited no difference in phenotypes compared with WT/PyMT mice.

We previously reported an inducible global epsin 1 and 2 double KO mouse model, tamoxifen-inducible Epn1 $1^{f / f l} E p n 2^{-/-} E R^{T 2}$-Cre mice on the C57BL/6J background $(22,24)$. Upon tamoxifen induction, the $\mathrm{ER}^{\mathrm{T} 2}$-driven Cre recombinase is ubiquitously expressed. To generate the inducible global epsin 1 and 2 double KO spontaneous breast cancer mouse model, we crossed Epn1 $1^{f / f l} E p n 2^{-/-} E R^{T 2}$-Cre mice with $M M T V-P y M T$ mice on the C57BL/6 background. The crosses resulted in Epn1 ${ }^{f / f l} E p n 2^{--} E R^{T 2}$-Cre MMTV-PyMT (iDKO/PyMT) mice. Similarly, for additional controls, we utilized Epn1 $1^{+/+} E p n 2^{-/-} E R^{T 2}-C r e$ MMTV-PyMT and Epn1 $1^{f / f l} E p n 2^{-/-} M M T V-P y M T$ littermates, both of which exhibited no difference in phenotypes compared with WT/ PyMT mice. To induce postnatal deletion, 4-hydroxytamoxifen was administered by i.p. injection ( $150 \mu \mathrm{g}$ per $30 \mathrm{~g}$ of body weight) at 6 weeks of age on alternate days for 2 weeks. To rule out the effect of 4-hydroxytamoxifen, we administered 4-hydroxytamoxifen to all animals, including the Cre-negative controls.

To rescue defective NF- $\kappa \mathrm{B}$ signaling, we crossed the $M E p C-D K O$ mouse with the R26Stop ${ }^{F L} I k k 2 c a$ (IKK2ca) mouse (61) on the C57BL/6 background purchased from the Jackson Laboratory (008242) to generate the $M E p C-D K O / I K K 2 c a^{f /+}$ mouse. An IKK2ca mouse harbors a 
constitutively activated $I K K 2 \mathrm{caf/+}$ transgene. Upon exposure to Cre recombinase, a floxed stop codon was removed, leading to the expression of the constitutively active IKK2 transgene and subsequent NF- $\mathrm{B}$ activation. The $M E p C-D K O / I K K 2 c a^{f l++}$ mouse was experimentally compared with $M E p C-D K O$ littermates.

Microarray analysis. RNA was collected from control and KD MDA231 cells using the RNeasy Mini Kit (Qiagen) according to the manufacturer's instructions. Gene expression profiles of the MDA231 cells with and without epsin 1 and 2 expression were determined using Agilent human GE $4 \times 44 \mathrm{k}$ microarrays (G4112F) following the manufacturer's instructions (G4140-90051). RNA samples and universal human reference RNA (Stratagene) were labeled with CTP-Cy5 and CTP-Сy3, respectively. The expression value of individual probes refers to the $\log _{2}(\mathrm{Cy} 5 / \mathrm{Cy} 3)$ ratio. The raw and normalized microarray data have been deposited in NCBI's Gene Expression Omnibus (GEO GSE63828).

Statistics. Group size was determined based on preliminary datasets. Statistical significance was determined using unpaired, 2-tailed parametric Student's $t$ test. One-way ANOVA with Tukey's multiple-comparisons test and 2-way ANOVA followed by Sidak's multiple-comparisons test were applied. A 95\% CI was considered for statistics and a $P$ value less than 0.05 was considered significant. All statistical analyses were performed using GraphPad Prism 7. All in vitro experiments were performed at least 3 times with similar results unless otherwise indicated.

Study approval. All animal procedures were approved by the IACUC of the Oklahoma Medical Research Foundation and Boston Children's Hospital.

\section{Author contributions}

$\mathrm{KS}, \mathrm{XC}$, and $\mathrm{HC}$ designed the experiments. KS primarily contributed to the identification of the in vitro molecular mechanism and XC primarily contributed to the in vivo data. YD contributed to UIM peptide therapy experiments and part of the molecular mechanism investigation. HW worked on part of the in vitro mechanism studies. SW provided technical support with mouse genotyping and colony maintenance. XC, YD, KS, and LY performed IP and Western blotting experiments. YW and YK performed the microarray analysis. UTS and KC performed GSEA of the TCGA BRCA. KS and XC performed the statistical and data analyses. AW shared HCl-019 (see Supplemental Methods). LX and DRB provided materials, animals, and/or suggestions. YL, BZ, SB, BW, KZ, ALW, and KAC provided technical support and/or comments. KS, $\mathrm{XC}$, and $\mathrm{HC}$ wrote the manuscript.

\section{Acknowledgments}

We thank the Huntsman Cancer Institute in Salt Lake City, Utah, for the use of the Preclinical Research Resource, which provided assistance in the derivation of patient-derived xenografts and animal services. This work was supported in part by NIH grants R01HL-093242, R01HL-118676, R01HL-130845, R01H-133216, R01HL-137229, and R01HL-146134; Established Investigator Award from the American Heart Association (AHA) to HC; AHA SDG grant 17SDG33410868 to HW; AHA SDG grant 17SDG33630161 to KS; Oklahoma Center for Advanced Science and Technology (OCAST) grants AR11-043 and HR14-056 and AHASDG grant12SDG8760002 to YD; OCAST grant HR09-116 and Department of Defense grant W81XWH-11-1-00226 to HC; grants from the Department of Defense (BC123187), Brewster Foundation, Breast Cancer Research Foundation, and the NIH (R01CA134519 and R01CA141062) to YK; and NIH grants R01HD083418, R01DK085691, and P01HL-085607 to LX.

Address correspondence to: Hong Chen, Vascular Biology Program, Boston Children's Hospital, Harvard Medical School, Boston, Massachusetts 02115, USA. Phone: 617.9196304; Email: hong.chen@childrens.harvard.edu.
1. Biswas DK, Dai SC, Cruz A, Weiser B, Graner E, Pardee AB. The nuclear factor kappa B (NF$\kappa \mathrm{B})$ : a potential therapeutic target for estrogen receptor negative breast cancers. Proc Natl Acad Sci U S A . 2001;98(18):10386-10391.

2. Biswas DK, et al. NF- $\kappa$ B activation in human breast cancer specimens and its role in cell proliferation and apoptosis. Proc Natl Acad Sci US A. 2004;101(27):10137-10142.

3. Liu M, et al. Nuclear factor- $\kappa B$ enhances ErbB2-induced mammary tumorigenesis and neoangiogenesis in vivo. Am J Pathol. 2009;174(5):1910-1920.

4. Huber MA, et al. NF- $\kappa B$ is essential for epithelial-mesenchymal transition and metastasis in a model of breast cancer progression. J Clin Invest. 2004;114(4):569-581.

5. Snyder M, Huang J, Huang XY, Zhang JJ. A signal transducer and activator of transcription 3. nucle-

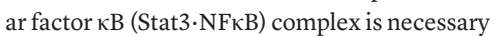
for the expression of fascin in metastatic breast cancer cells in response to interleukin (IL)-6 and tumor necrosis factor (TNF)- $\alpha$. J Biol Chem. 2014;289(43):30082-30089.

6. Qiao Y, He H, Jonsson P, Sinha I, Zhao C, Dahlman-Wright K. AP-1 is a key regulator of proinflammatory cytokine $\mathrm{TNF} \alpha$-mediated triple-negative breast cancer progression. J Biol
Chem. 2016;291(10):5068-5079.

7. Schneider-Brachert W, et al. Compartmentalization of TNF receptor 1 signaling: internalized TNF receptosomes as death signaling vesicles. Immunity. 2004;21(3):415-428.

8. DiDonato JA, Hayakawa M, Rothwarf DM, Zandi E, Karin M. A cytokine-responsive IкB kinase that activates the transcription factor NF-кB. Nature. 1997;388(6642):548-554.

9. Rothwarf DM, Zandi E, Natoli G, Karin M. IKK- $\gamma$ is an essential regulatory subunit of the I $\mathrm{B}$ kinase complex. Nature. 1998;395(6699):297-300.

10. Zandi E, Rothwarf DM, Delhase M, Hayakawa M, Karin M. The IкB kinase complex (IKK) contains two kinase subunits, IKKalpha and IKKbeta, necessary for I $\mathrm{B}$ phosphorylation and NF- $\kappa \mathrm{B}$ activation. Cell. 1997;91(2):243-252.

11. Abbott DW, Wilkins A, Asara JM, Cantley LC. The Crohn's disease protein, NOD2, requires RIP2 in order to induce ubiquitinylation of a novel site on NEMO. Curr Biol. 2004;14(24):2217-2227.

12. Sebban-Benin $\mathrm{H}$, et al. Identification of TRAF6-dependent NEMO polyubiquitination sites through analysis of a new NEMO mutation causing incontinentia pigmenti. $\mathrm{Hum} \mathrm{Mol}$ Genet. 2007;16(23):2805-2815.

13. Ni CY, et al. Cutting edge: K63-linked poly- ubiquitination of NEMO modulates TLR signaling and inflammation in vivo. J Immunol. 2008;180(11):7107-7111.

14. Tokunaga $\mathrm{F}$, et al. Involvement of linear polyubiquitylation of NEMO in NF- $\kappa \mathrm{B}$ activation. Nat Cell Biol. 2009;11(2):123-132.

15. Fujita $\mathrm{H}$, et al. Mechanism underlying I $\mathrm{B}$ kinase activation mediated by the linear ubiquitin chain assembly complex. Mol Cell Biol. 2014;34(7):1322-1335.

16. Bloor $S$, et al. Signal processing by its coil zipper domain activates IKK $\gamma$. Proc Natl Acad Sci U S A. 2008;105(4):1279-1284.

17. Rahighi S, et al. Specific recognition of linear ubiquitin chains by NEMO is important for NF-кB activation. Cell. 2009;136(6):1098-1109.

18. Catici DA, Horne JE, Cooper GE, Pudney CR. Polyubiquitin drives the molecular interactions of the NF- $\mathrm{BB}$ essential modulator (NEMO) by allosteric regulation. J Biol Chem. 2015;290(22):14130-14139.

19. May MJ, Marienfeld RB, Ghosh S. Characterization of the IкB-kinase NEMO binding domain. J Biol Chem. 2002;277(48):45992-46000.

20. Chen H, et al. Epsin is an EH-domain-binding protein implicated in clathrin-mediated endocytosis. Nature. 1998;394(6695):793-797.

21. Chen H, De Camilli P. The association of epsin 
with ubiquitinated cargo along the endocytic pathway is negatively regulated by its interaction with clathrin. Proc Natl Acad Sci U S A. 2005;102(8):2766-2771.

22. Tessneer KL, et al. Endocytic adaptor protein epsin is elevated in prostate cancer and required for cancer progression. ISRN Oncol. 2013;2013:420597.

23. Coon BG, Burgner J, Camonis JH, Aguilar RC. The epsin family of endocytic adaptors promotes fibrosarcoma migration and invasion. J Biol Chem. 2010;285(43):33073-33081.

24. Pasula S, et al. Endothelial epsin deficiency decreases tumor growth by enhancing VEGF signaling. JClin Invest. 2012;122(12):4424-4438.

25. Ko G, et al. Selective high-level expression of epsin 3 in gastric parietal cells, where it is localized at endocytic sites of apical canaliculi. Proc Natl Acad Sci U S A. 2010;107(50):21511-21516.

26. Chang B, et al. Epsin is required for Dishevelled stability and Wnt signalling activation in colon cancer development. Nat Commun. 2015;6:6380.

27. Spradling KD, McDaniel AE, Lohi J, Pilcher BK. Epsin 3 is a novel extracellular matrix-induced transcript specific to wounded epithelia. J Biol Chem. 2001;276(31):29257-29267.

28. Chen $\mathrm{H}$, et al. Embryonic arrest at midgestation and disruption of Notch signaling produced by the absence of both epsin 1 and epsin 2 in mice. Proc Natl Acad Sci U S A. 2009;106(33):13838-13843.

29. Peltzer N, et al. LUBAC is essential for embryogenesis by preventing cell death and enabling haematopoiesis. Nature. 2018;557(7703):112-117.

30. Tessneer KL, et al. Genetic reduction of vascular endothelial growth factor receptor 2 rescues aberrant angiogenesis caused by epsin deficiency. Arterioscler Thromb Vasc Biol. 2014;34(2):331-337.

31. Wendland B. Epsins: adaptors in endocytosis? Nat Rev Mol Cell Biol. 2002;3(12):971-977.

32. Tanner N, Kleifeld O, Nachman I, Prag G. Remodeling membrane binding by monoubiquitylation. Biomolecules. 2019;9(8):E325.

33. Wu H, et al. Epsin deficiency promotes lymphangiogenesis through regulation of VEGFR3 degradation in diabetes. J Clin Invest. 2018;128(9):4025-4043.

34. Liu X, et al. Temporal and spatial regulation of epsin abundance and VEGFR3 signaling are required for lymphatic valve formation and function. Sci Signal. 2014;7(347):ra97.
35. Kharman-Biz A, Gao H, Ghiasvand R, Haldosen LA, Zendehdel K. Expression of the three components of linear ubiquitin assembly complex in breast cancer. PLoS One. 2018;13(5):e0197183.

36. Dong Y, et al. Motif mimetic of epsin perturbs tumor growth and metastasis. JClin Invest. 2015;125(12):4349-4364.

37. Rahman HNA, et al. Selective targeting of a novel epsin-VEGFR2 interaction promotes VEGF-mediated angiogenesis. Circ Res. 2016;118(6):957-969.

38. Conner JR, Smirnova II, Moseman AP, Poltorak A. IRAK1BP1 inhibits inflammation by promoting nuclear translocation of NF-kB p50. Proc Natl Acad SciUS A. 2010;107(25):11477-11482.

39. Micheau O, Tschopp J. Induction of TNF receptor I-mediated apoptosis via two sequential signaling complexes. Cell. 2003;114(2):181-190.

40. Peltzer N, et al. HOIP deficiency causes embryonic lethality by aberrant TNFR1-mediated endothelial cell death. Cell Rep. 2014;9(1):153-165.

41. Schütze S, Tchikov V, Schneider-Brachert W. Regulation of TNFR1 and CD95 signalling by receptor compartmentalization. Nat Rev Mol Cell Biol. 2008;9(8):655-662.

42. Kondratyev M, et al. Gamma-secretase inhibitors target tumor-initiating cells in a mouse model of ERBB2 breast cancer. Oncogene. 2012;31(1):93-103.

43. Chen ZJ. Ubiquitin signalling in the NF- $\mathrm{KB}$ pathway. Nat Cell Biol. 2005;7(8):758-765.

44. Emmerich $\mathrm{CH}$, et al. Activation of the canonical IKK complex by K63/M1-linked hybrid ubiquitin chains. Proc Natl Acad Sci U S A. 2013;110(38):15247-15252.

45. Polo S, et al. A single motif responsible for ubiquitin recognition and monoubiquitination in endocytic proteins. Nature. 2002;416(6879):451-455.

46. Oldham CE, Mohney RP, Miller SL, Hanes RN, O'Bryan JP. The ubiquitin-interacting motifs target the endocytic adaptor protein epsin for ubiquitination. Curr Biol. 2002;12(13):1112-1116.

47. Ea CK, Deng L, Xia ZP, Pineda G, Chen ZJ. Activation of IKK by TNF $\alpha$ requires site-specific ubiquitination of RIP1 and polyubiquitin binding by NEMO. Mol Cell. 2006;22(2):245-257.

48. Witt A, Vucic D. Diverse ubiquitin linkages regulate RIP kinases-mediated inflammatory and cell death signaling. Cell Death Differ.
2017;24(7):1160-1171.

49. Haas TL, et al. Recruitment of the linear ubiquitin chain assembly complex stabilizes the TNF-R1 signaling complex and is required for TNF-mediated gene induction. Mol Cell. 2009;36(5):831-844.

50. Gerlach B, et al. Linear ubiquitination prevents inflammation and regulates immune signalling. Nature. 2011;471(7340):591-596.

51. Tokunaga F, et al. SHARPIN is a component of the NF- $\mathrm{kB}$-activating linear ubiquitin chain assembly complex. Nature. 2011;471(7340):633-636.

52. Habelhah H. Emerging complexity of protein ubiquitination in the NF- $\mathrm{kB}$ pathway. Genes Cancer. 2010;1(7):735-747.

53. Hrdinka M, Gyrd-Hansen M. The Met1-linked ubiquitin machinery: emerging themes of (de) regulation. Mol Cell. 2017;68(2):265-280.

54. Jun JC, et al. Innate immune-directed NF- $\mathrm{\kappa B}$ signaling requires site-specific NEMO ubiquitination. Cell Rep. 2013;4(2):352-361.

55. Vlantis K, et al. Constitutive IKK2 activation in intestinal epithelial cells induces intestinal tumors in mice. JClin Invest. 2011;121(7):2781-2793.

56. Jimi E, et al. Selective inhibition of NF- $\mathrm{KB}$ blocks osteoclastogenesis and prevents inflammatory bone destruction in vivo. Nat Med. 2004;10(6):617-624.

57. May MJ, D’Acquisto F, Madge LA, Glöckner J, Pober JS, Ghosh S. Selective inhibition of NF- $\mathrm{\kappa B}$ activation by a peptide that blocks the interaction of NEMO with the IKB kinase complex. Science. 2000;289(5484):1550-1554.

58. DeRose YS, et al. Tumor grafts derived from women with breast cancer authentically reflect tumor pathology, growth, metastasis and disease outcomes. Nat Med. 2011;17(11):1514-1520.

59. DeRose YS, et al. Patient-derived models of human breast cancer: protocols for in vitro and in vivo applications in tumor biology and translational medicine. Curr Protoc Pharmacol. 2013; Chapter 14:Unit14.23.

60. Wagner KU, et al. Cre-mediated gene deletion in the mammary gland. Nucleic Acids Res. 1997;25(21):4323-4330.

61. Calado DP, et al. Constitutive canonical NF- $\mathrm{\kappa B}$ activation cooperates with disruption of BLIMP1 in the pathogenesis of activated B celllike diffuse large cell lymphoma. Cancer Cell. 2010;18(6):580-589. 